\title{
Truthful resolutions: A new perspective on false-answer sensitivity*
}

\author{
Nadine Theiler \\ University of Amsterdam
}

\author{
Floris Roelofsen \\ University of Amsterdam
}

\author{
Maria Aloni \\ University of Amsterdam
}

\begin{abstract}
Responsive verbs like know embed both declarative and interrogative complements. Standard accounts of such verbs are reductive: they assume that whether an individual stands in a knowledge- $w h$ relation to a question is determined by whether she stands in a knowledge-that relation to some answer to the question. George (2013) observed that knowledge-wh, however, not only depends on knowledge-that but also on false belief - a fact that reductive accounts can't capture. We develop an account that is not reductive but uniform: it assumes a single entry for interrogative-embedding and declarative-embedding uses of a responsive verb. The key insight that allows us to capture the false-belief dependency of knowledgewh is that verbs like know are sensitive to both true and false answers to the embedded question. Formally, this is achieved through a novel, fine-grained way of representing the meaning of a clausal complement in terms of so-called truthful resolutions. The resulting analysis gives us a unifying perspective, under which false-answer sensitivity comes out as a general characteristic common to all levels of exhaustivity.
\end{abstract}

Keywords: embedded questions, responsive verbs, false-answer sensitivity, reducibility of knowledge- $w h$, mention-some readings

\section{Introduction}

This paper gives an analysis of clausal complements and the verbs that take such complements, focusing on two concrete examples, know and be certain. These verbs are responsive, i.e., they can embed both interrogative complements, as in (1a) and declarative complements, as in (1b).

* We are very grateful to Boban Arsenijevic, Lucas Champollion, Ivano Ciardelli, Alexandre Cremers, Paul Egré, Donka Farkas, Jeroen Groenendijk, Benjamin Spector, and Wataru Uegaki for discussion of the ideas presented here and closely related issues. We also thank the SALT reviewers and participants for insightful questions and comments. Finally, we gratefully acknowledge financial support from the Netherlands Organisation for Scientific Research (NWO). 
Truthful resolutions

(1) a. John knows/is certain which spices go into a green curry.

b. John knows/is certain that cumin goes into a green curry.

In the literature, responsive verbs are often treated by assuming two distinct lexical items for each verb, one for its interrogative-embedding and one for its declarativeembedding use (see, e.g., Karttunen 1977; George 2011; Spector \& Egré 2015). For instance, know would have two entries, know $_{i}$ and know ${ }_{d}$, taking interrogative and declarative complements, respectively. Under this approach, the hope is that the two entries can be systematically connected. In particular, a substantial effort has been made to find a general procedure that, given the declarative entry $V_{d}$ for any verb $V$, delivers the interrogative entry $V_{i}$ for $V$ (see in particular George 2011; Spector \& Egré 2015). Since this amounts to reducing the interrogative entry to the declarative one, accounts that follow this strategy are called reductive.

\subsection{George's challenge as a problem for reductive accounts}

George $(2011,2013)$ pointed out a problem for a reductive treatment of know: declarative knowledge insufficiently determines interrogative knowledge. As evidence, he provides a scenario in which two individuals, $a$ and $b$, have exactly the same declarative knowledge (that is, for all propositions $p, a$ knows $_{d} p$ iff $b$ knows $_{d} p$ ) and yet $a$ and $b$ come apart in their interrogative knowledge (i.e., there is a question $Q$ such that $a$ knows $_{i} Q$ but $b$ doesn't know $_{i} Q$ ). More concretely, assume that there are two shops, Newstopia and Paperworld, but only one of them, namely Newstopia, sells Italian newspapers. Both Janna and Rupert know that Newstopia sells Italian newspapers. Janna doesn't have beliefs concerning the availability of such newspapers elsewhere, while Rupert falsely believes that they are also sold at Paperworld. Assuming that Janna and Rupert have no further beliefs, we thus find that, for all propositions $p$, Janna knows $_{d} p$ iff Rupert knows $d p$. This is the case since Rupert's belief about Paperworld is not something he knows ${ }_{d}$, quite simply because it is false. In this scenario, there is a reading under which (2) is judged true, while (3) is judged false-meaning that Janna and Rupert have different interrogative knowledge.

(2) Janna knows where one can buy an Italian newspaper.

(3) Rupert knows where one can buy an Italian newspaper.

One way of thinking about what goes wrong here, George indicates, is to realize that interrogative knowledge does not only depend on true declarative knowledge, but also on possibly false beliefs. Crucially, however, it is not just any false belief that plays a role, but only those false beliefs that would lead the individual to falsely answer the embedded interrogative. In the above scenario, for instance, (2) will still be judged true if Janna has wrong beliefs that have no bearing on the embedded interrogative, e.g., if she mistakenly believes that Barcelona is the capital of Spain. 


\subsection{False-answer sensitivity across different levels of exhaustivity}

In order to deal with George's challenge, we need to capture the meaning of interrogative complements and their embedding verbs in a way that is sensitive not just to true but also to false answers of the complement. It is important to observe that this differs from standard accounts of interrogative complements. So, let us take a moment to recap the traditional picture.

Traditionally, three readings are distinguished for sentences like (4) below: the strongly exhaustive (SE), weakly exhaustive (WE) and mention-some (MS) reading. Assume a domain of discourse $D$, assume John knows that the domain of discourse is $D$, and assume the set of answers to the question Who called? is $A=\{d$ called $\mid d \in D\}$. Then, for (4) to be true under an SE reading, John needs to know of every true answer $p \in A$ that $p$ is true and of every false answer $p \in A$ that $p$ is false. Under a WE reading, it suffices if John knows of every true answer $p \in A$ that $p$ is true; and under an MS reading John only needs to know of one true answer $p \in A$ that $p$ is true.

John knows who called.

Under the traditional view, false answers are hence only relevant for SE readings, not for WE or MS readings. Recently, however, it has been argued under the heading of false-answer sensitivity (FA sensitivity) that false answers also play a role for readings weaker than SE. We have already seen the argument in favour of FA-sensitive MS readings, namely George's challenge. ${ }^{1}$ The Italian-newspaper complement used there is a typical MS example: (2) is true by virtue of Janna knowing just one true answer of the complement.

The argument for FA-sensitive WE readings, aka intermediate exhaustive (IE) readings, ${ }^{2}$ has been given by Spector (2005), whose empirical claims have since been corroborated experimentally by Cremers \& Chemla (2016) and have influenced various recent theoretical proposals (Klinedinst \& Rothschild 2011; Spector \& Egré 2015; Uegaki 2015; Cremers 2016). ${ }^{3}$

However, while these recent theories do incorporate FA sensitivity, they each limit their considerations to just one specific level of exhaustivity: George (2011, 2013) focuses exclusively on FA-sensitive MS readings, while Spector (2005), Klinedinst \& Rothschild (2011), Uegaki (2015) and Spector \& Egré (2015) focus on IE readings. In contrast, our goal will be to give a general characterization of FA sensitivity that applies uniformly across the different levels of exhaustive strength.

1 Such readings have also already been considered in Groenendijk \& Stokhof (1984).

2 This term was coined by Klinedinst \& Rothschild (2011).

3 The reason why we presented George's and not Spector's argument, although the latter precedes the former, is that George explicitly used his scenario to demonstrate the "non-reducibility" of interrogative knowledge — an aspect in which we are interested here. 
Truthful resolutions

\subsection{Our approach: A uniform semantics}

We will approach the problem of responsive verbs from a different angle, giving not a reductive, but a uniform account. That is, we won't derive the interrogativeembedding entry from the declarative-embedding one, or vice versa, but instead we assume only one lexical item for each responsive verb, which can apply to declarative and interrogative complements alike. This way, we obtain a maximally parsimonious account and bring out a maximally close connection between the two different uses of responsive verbs. George's challenge, as will be shown, does not arise for our approach.

In the course of this paper, we will see that the effectiveness of the account rests on two cornerstones. Firstly, the meaning of the complement clause is modelled as a set of so-called truthful resolutions, a fine-grained meaning representation that synthesizes true and false answers. This first cornerstone is essential for deriving FA sensitivity. Secondly, the embedding verbs are sensitive to the semantic properties that distinguish interrogative from declarative complements. This sensitivity gives rise to different semantic effects, depending on which kind of complement the verb takes.

The remainder of the paper is structured as follows. First, Section 2 briefly reviews the basics of the type-theoretic inquisitive semantics framework we adopt. In Section 3, we turn to the account proper, first laying out some general assumptions which apply to both declarative and interrogative complements, then zooming in on interrogative complements in Section 3.1 and on declarative complements in Section 3.2. Finally, Section 4 concludes.

\section{Semantic framework}

Our account will be couched in the framework of inquisitive semantics (Ciardelli, Groenendijk \& Roelofsen 2013, 2015), more specifically in the type-theoretical inquisitive semantics developed in Ciardelli, Roelofsen \& Theiler (2016). It is worth pointing out, however, that our ideas would in principle also be compatible with a Hamblin-style semantics.

\subsection{Sentence meanings in inquisitive semantics}

In traditional semantic frameworks, the meaning of a sentence $\varphi$ is construed as a proposition, i.e., a set of possible worlds. This captures the informative content conveyed by $\varphi$. Inquisitive semantics generalizes this notion of meaning to capture not just informative, but also inquisitive content, i.e., the issue raised by $\varphi$. To achieve this, the meaning of a sentence is construed as a set of propositions. When a speaker utters a sentence with meaning $P$, she is taken to raise an issue whose 
resolution requires establishing one of the propositions in $P$, while at the same time providing the information that the actual world is contained in the union of these propositions, $\bigcup P$. We refer to the elements of $P$ as the resolutions of the sentence.

Sentence meanings in inquisitive semantics are downward closed: if $p \in P$ and $q \subset p$, then also $q \in P$. This captures the intuition that, if a proposition $p$ resolves a given issue, then any stronger proposition $q \subset p$ will also resolve that issue. Finally, the inconsistent proposition, $\emptyset$, is assumed to resolve any issue. So, any sentence meaning contains $\emptyset$ and is thus non-empty.

Definition 1 (Sentence meanings in inquisitive semantics). A sentence meaning in inquisitive semantics is a non-empty, downward closed set of propositions.

The maximal elements of $P$ are referred to as the alternatives in $P$. We write alt $(P)$ for the set of all alternatives in $P$, alt ${ }_{w}(P)$ for the set of those alternatives that are true in $w$, and alt $_{w}^{*}(P)$ for those that are false in $w$. In depicting a sentence meaning, we generally only depict its alternatives. Finally, info $(P)=\bigcup P$ is referred to as the informative content of $P$, and a sentence with meaning $P$ is said to be true in a world $w$ iff $w \in \operatorname{info}(P)$.

Definition 2 (Alternatives, informative content, and truth). For any sentence meaning $P$ and any world $w$ :

- $\operatorname{alt}(P):=\{p \in P \mid$ there is no $q \in P$ such that $p \subset q\}$

- $\operatorname{alt}_{w}(P):=\{p \in \operatorname{alt}(P) \mid w \in p\}$

- $\operatorname{alt}_{w}^{*}(P):=\{p \in \operatorname{alt}(P) \mid w \notin p\}$

- $\operatorname{info}(P):=\bigcup P$

- A sentence with meaning $P$ is true in $w$ iff $w \in$ info $(P)$.

To illustrate these notions, consider the following three sentences:
a. Did Amy leave?
b. Who left?
c. Amy left.

These sentences may be assigned the meanings depicted in Figure 1, where $w_{a b}$ and $w_{a}$ are worlds where Amy left, $w_{b}$ and $w_{\emptyset}$ are worlds where Amy didn't leave, $w_{a b}$ and $w_{b}$ are worlds where Bill left, and $w_{a}$ and $w_{\emptyset}$ are worlds where Bill didn't leave. The shaded rectangles are the alternatives contained in the given meanings. By downward closure, all propositions contained in one of these alternatives are also included in the meanings of the sentences. 

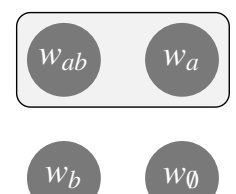

$w_{b}$

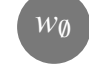

(a) Amy left.

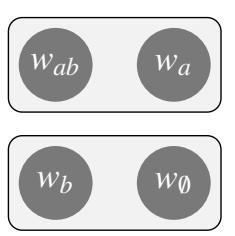

(b) Did Amy leave?

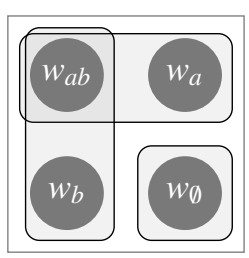

(c) Who left?[-exh]

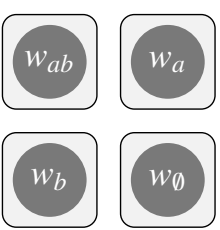

(d) Who left? $[+e x h]$

Figure 1 The meanings of some simple sentences in inquisitive semantics.

We will not discuss each example in detail here, but only point out certain features. Most importantly, observe that the declarative meaning in Figure 1a, contains a single alternative, while the interrogative meanings in Figure 1b-1d each contain several alternatives, and that each interrogative meaning covers the entire logical space. Also note that the alternatives in an interrogative meaning correspond (roughly) to what are called answers in, e.g., a Hamblin-style theory of questions. Finally, we assume that the $w h$-interrogative Who left? has two possible readings, an exhaustive and a non-exhaustive one. On the exhaustive reading, it has the meaning in Figure 1d and on the non-exhaustive reading the one in Figure 1c. An exhaustive interrogative meaning partitions the logical space, while a non-exhaustive interrogative meaning typically contains overlapping alternatives.

\subsection{Informative and inquisitive sentences}

As mentioned above, an interrogative sentence meaning covers the entire logical space $W$. Since the information that is provided by such sentences is trivial, we call them non-informative. Conversely, a sentence with meaning $P$ is called informative just in case $\operatorname{info}(P) \neq W$.

On the other hand, in the case of declaratives, the inquisitive content of the sentence is trivial, in the sense that the issue that is raised in uttering the sentence is already resolved by the information provided; no further information is required. We call such sentences non-inquisitive. Conversely, a sentence with meaning $P$ is called inquisitive just in case it doesn't provide enough information to resolve the issue that it raises, i.e., iff info $(P) \notin P$.

It is easy to see from a diagram whether the depicted sentence is inquisitive. A sentence is inquisitive iff its meaning contains at least two alternatives. ${ }^{4}$ For

4 Strictly speaking, this only holds under the assumption that there are finitely many worlds-but this is a safe assumption to make for all the examples to be considered in this paper. 


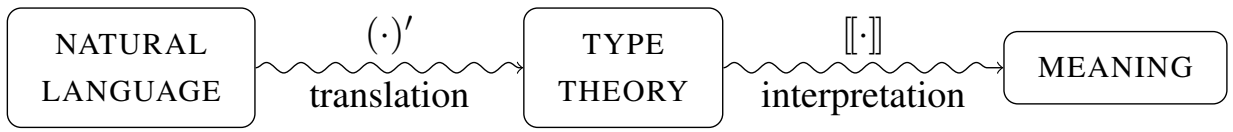

Figure 2 Two-step setup for deriving sentence meanings

instance, the meaning in Figure $1 \mathrm{~b}$ contains two alternatives, i.e., the interrogative Did Amy leave? is inquisitive, while the meaning in Figure 1a contains only one alternative, i.e., the declarative Amy left is non-inquisitive.

\subsection{Composing meanings}

We will now give a very quick introduction to type-theoretic inquisitive semantics and see how inquisitive sentence meanings can be derived compositionally. For a more systematic introduction, the reader is referred to Ciardelli et al. (2016).

We adopt a standard two-step approach, summarized in Figure 2. In the first step, we translate a natural language expression into a type-theoretic language, by translating every lexical item into a certain expression and combining these expressions via standard function application and abstraction. We write $(\alpha)^{\prime}$ for the translation of a natural language expression $\alpha$. In the second step, type-theoretic expressions are interpreted relative to a model $\mathscr{M}$ and an assignment $g$.

The type theory we assume is two-sorted, with basic types $e, s$, and $t$, for individuals, worlds, and truth values, respectively. Since sentence meanings are construed as sets of propositions, sentences are taken to be of type $\langle\langle s, t\rangle, t\rangle$, which we abbreviate as $T$. From this, we can reverse engineer the types that should be assigned to various kinds of sub-sentential expressions:

John : $e$

walks : $\langle e, T\rangle$ likes : $\langle e,\langle e, T\rangle\rangle$

not : $\langle T, T\rangle$ and : $\langle T,\langle T, T\rangle\rangle$

somebody : $\langle\langle e, T\rangle, T\rangle$

More concretely, the meaning of a sentence like John walks is the set of those propositions $p$ that exclusively consist of worlds $w$ in which John walks:

$$
\text { (John walks) }{ }^{\prime}=\lambda p . \forall w \in p: W(j)(w)
$$

To obtain the above sentence meaning, the verb walks should express a function that takes an individual $x$ and yields the set of propositions $p$ such that $x$ walks in every world in $p$ :

$$
\text { walks }^{\prime}=\lambda x . \lambda p . \forall w \in p: W(x)(w)
$$




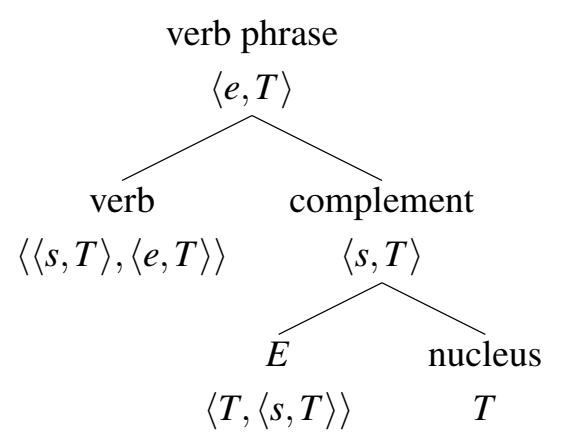

(a) Global structure of complement constructions

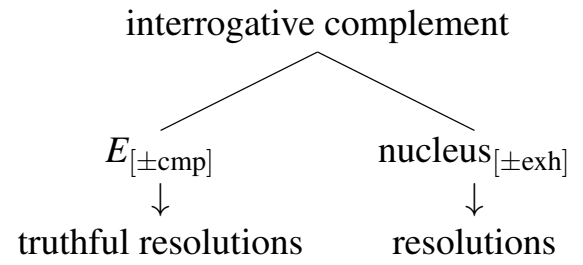

(b) Structure of interrogative complements

Figure 3 Assumptions about the structure of complement constructions

\section{A truthful resolution semantics for complement clauses}

We will now present our account proper. We start by laying out some general assumptions that are common to both interrogative and declarative complements, and then zoom in on either kind of complement in turn.

We assume that complement constructions have the global structure in Figure 3a. Declarative and interrogative complements both involve an embedding operator $E$, which adjoins to a clause that we will call the nucleus. The nucleus has the same semantic properties - for our current purposes — as a declarative respectively interrogative root clause. The meaning of a root clause, as discussed in Section 2, is the set of resolutions of the issue raised by the clause. Hence, the meaning of the nucleus is the set of resolutions of the issue expressed by the nucleus; it has type $T$. Notice that the nucleus meaning is thus a world-independent object.

We can think of $E$ as intermediating between nucleus meaning and embedding verb by turning the world-independent nucleus meaning into a world-sensitive object. More concretely, $E$ takes a set of resolutions as its input and returns a function that maps every world $w$ to the set of truthful resolutions at $w$. The relevant terminology is summarized in Figure 3b.

\subsection{Interrogative complements}

We assume that interrogative nuclei, just as interrogative root clauses, are interpreted either exhaustively or non-exhaustively (see Section 2). We write [nucleus $[+\mathrm{exh}]]$

for the exhaustive and [nucleus $[-$ exh $]]$ for the non-exhaustive interpretation of the 
nucleus. ${ }^{5}$ The $E$ operator is parameterized as well, namely for completeness, a feature to be made precise below. As we will see, a combination of these two parameters allows us to capture a three-way distinction between MS, WE and SE readings, all of them with FA sensitivity.

\subsubsection{The $E$ operator: From resolutions to truthful resolutions}

As we saw in Section 2, in order to count as a resolution of some issue, a proposition has to provide enough information to resolve this issue. In the case of a whinterrogative, for instance, the minimally informative propositions that provide enough information to resolve the issue raised by the interrogative are the Hamblin answers. Naturally, however, if a proposition $p$ resolves an issue $P$, then any more informative proposition $q \subset p$ will resolve $P$ as well. This is the reason why sentence meanings in inquisitive semantics, which are taken to be sets of resolutions, are downward-closed. For example, the meaning of a $w h$-interrogative in inquisitive semantics can be obtained by taking the downward closure of the corresponding meaning in Hamblin semantics.

However, on our account, the meaning of a complement is not represented as a set of resolutions, but as a function from worlds to sets of truthful resolutions. As suggested by their name, truthful resolutions are still resolutions, but in addition they have to fulfil two requirements: they need to be consistent, and they mustn't provide false information w.r.t. the given issue.

Formally, this latter condition amounts to the following. Let $P$ be a sentence meaning. Then, a resolution $p \in P$ provides false information w.r.t. $P$ in world $w$ iff $p$ entails an alternative in $P$ which is false in $w$. Conversely, $p$ provides no false information w.r.t. $P$ in $w$ iff $p$ entails no alternative in $P$ that is false in $w$. For instance, assume that Amy and Bill are the only individuals in the domain and that only Amy left (in the diagrams in Figure 1, this means the actual world is $w_{a}$ ). Consider the sentence meaning $P=\left[\left[\right.\right.$ who left $\left.\left._{[-\mathrm{exh}]}\right]\right]$, which contains one alternative that is true in $w_{a}$ (Amy left) and two alternatives that are false in $w_{a}$ (Bill left; neither Amy nor Bill left). Let $p$ be the proposition that Amy and Bill left. Since $p$ entails the alternative that Bill left and this alternative is false in $w_{a}, p$ provides false information w.r.t. $P$ and is therefore not a truthful resolution.

We hence arrive at the following definition of truthful resolutions, where the truthfulness requirement corresponds to condition (iii) (recall that alt ${ }_{w}^{*}(P)$ denotes the set of alternatives in $P$ that are false in $w$ ).

5 For proposals on how to derive these two interpretations compositionally, in a way that is compatible with the present account of embedding, see Theiler (2014) and Champollion, Ciardelli \& Roelofsen (2015). 
Truthful resolutions

Definition 3 (Truthful resolution). Let $P$ be a sentence meaning and $w$ a possible world. A proposition $p$ is a truthful resolution of $P$ in $w$ iff:

(i) $p$ is a resolution of $P$,

(ii) $p$ is consistent,

(iii) $p$ does not entail any alternative in $\operatorname{alt}_{w}^{*}(P)$.

We further distinguish between truthful resolutions simpliciter and complete truthful resolutions. The former, as follows from clause (i) and (iii) in Definition 3, entail at least one true alternative, while the latter entail all true alternatives.

Definition 4 (Complete truthful resolution). Let $P$ be a sentence meaning and $w$ a possible world. A proposition $p$ is a complete truthful resolution of $P$ in $w$ iff:

(i) $p$ is a truthful resolution of $P$ in $w$,

(ii) $p$ entails all alternatives in alt $_{w}(P)$.

For example, consider a scenario in which there are three people, Amy, Bill and Clara. Assume that in world $w$ Amy and Bill left, but Clara didn't. Again, let $P=\left[\left[\right.\right.$ who left $\left.\left._{[-\mathrm{exh}]}\right]\right]$. Then, the proposition that Amy left, the proposition that Bill left, and the proposition that both of them left are all truthful resolutions of $P$ in $w$. The proposition that both of them left is additionally a complete truthful resolution of $P$ in $w$. The proposition that Amy, Bill and Clara left, on the other hand, is not a truthful resolution of $P$ in $w$ because it entails the false alternative that Clara left.

However, here is an interesting twist. In the above scenario, assume furthermore that it is Monday. Consider the proposition $p$ that Amy left and that it is Tuesday. Clearly, $p$ is false. Nonetheless, this proposition counts as a truthful resolution since it provides true information w.r.t. the issue of who left and the false information that it provides, namely that it is Tuesday, is not relevant w.r.t. the issue of who left.

Also observe that, once we make the step from resolutions to truthful resolutions, we are not dealing with downward-closed sets anymore: although the proposition that Amy, Bill and Clara left is a subset of the proposition that Amy left, only the latter is a truthful resolution of $P$ in $w$.

To get a more visual understanding of what this restricted version of downward closedness amounts to, consider Figure 4. Let $p$ be the proposition that Italian newspapers are sold at Newstopia and $q$ the proposition that they are sold at Paperworld. The actual world, $w_{0}$, is located in $p$, but not in $q$ since only Newstopia stocks Italian newspapers. Let us reflect on which propositions in the picture count as truthful resolutions of where can one get an Italian newspaper in $w_{0}$. Clearly, $p$ is a truthful resolution, or, to be more precise, the minimally informative truthful 


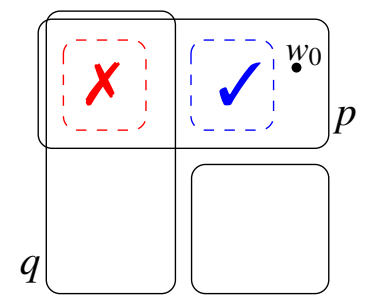

Figure 4 Restricted downward closedness

resolution. More interesting, however, is the question which subsets of $p$ are truthful resolutions and which are not. To begin with, all true propositions entailing $p$ are automatically truthful resolutions because they are consistent resolutions and cannot entail a false alternative. On the other hand, with false propositions that entail $p$, we have to distinguish two cases. First, let $r$ be the proposition that both Newstopia and Paperworld sell Italian newspapers (the crossed-out proposition in the diagram). Since $r$ entails $q$, it does not count as a truthful resolution. Second, let $r^{\prime}$ be some other consistent proposition such that $r^{\prime} \subseteq p$, but $r^{\prime} \nsubseteq q$ (the one with a tick mark in the diagram). Then, $r^{\prime}$ does not entail any alternative that is false in $w_{0}$. Hence, although both $r$ and $r^{\prime}$ are false, $r^{\prime}$ counts as a truthful resolution in $w_{0}$ whereas $r$ doesn't.

The insight to take away is that a truthful resolution need not be true; it only must not provide false information w.r.t. the issue. Truthful resolutions hence embody a notion of truth that is radically relativized to a given issue.

We now turn to defining the $E$ operator. When applied to a sentence meaning $P$ (for our purposes the nucleus meaning), this operator yields a function mapping every world $w$ to the set of (complete) truthful resolutions of $P$ in $w$. Formally, we can characterize $E$, which comes in a complete and a non-complete variant, as follows. ${ }^{6}$

Definition 5 (The $E$ operator).

$$
E_{[-\mathrm{cmp}]}:=\lambda P_{T} \cdot \lambda w \cdot \lambda p \cdot\left(\begin{array}{c}
p \in P \wedge p \neq \emptyset \wedge \\
\neg \exists q \in \mathrm{alt}_{w}^{*}(P) \cdot p \subseteq q
\end{array}\right)
$$

6 These two variants are formally quite different. The computation carried out by $E_{[-\mathrm{cmp}]}$ is an innocent type-shift, i.e., if we have a function $f=E_{[-\mathrm{cmp}]}(P)$, we can retrieve the set $P$ from $f$, since $P=\bigcup_{w \in W} f(w) \cup\{\emptyset\}$. In contrast, retrieving $P$ from $f=E_{[+\mathrm{cmp}]}(P)$ is not always possible. Hence, the computation carried out by $E_{[+\mathrm{cmp}]}$ is not an innocent type-shift. 
Truthful resolutions

$$
E_{[+\mathrm{cmp}]}:=\lambda P_{T} \cdot \lambda w \cdot \lambda p \cdot\left(\begin{array}{c}
p \in P \wedge p \neq \emptyset \wedge \\
\neg \exists q \in \mathrm{alt}_{w}^{*}(P) \cdot p \subseteq q \wedge \\
\forall q \in \mathrm{alt}_{w}(P) \cdot p \subseteq q
\end{array}\right)
$$

For an illustration of the functions that $E$ yields, consider the examples below, which show the result of applying this operator to typical question meanings. Again, observe that sets of truthful resolutions are not downward-closed. For instance,

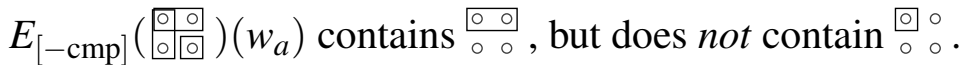

Further observe that, if $E$ applies to an exhaustive nucleus meaning $P, E_{[+\mathrm{cmp}]}(P)$ and $E_{[-\mathrm{cmp}]}(P)$ coincide. This is the case because, if $P$ is exhaustive, then alt ${ }_{w}(P)$ is a singleton set for every $w$, which means that any truthful resolution in $w$ is automatically a complete truthful resolution in $w$.

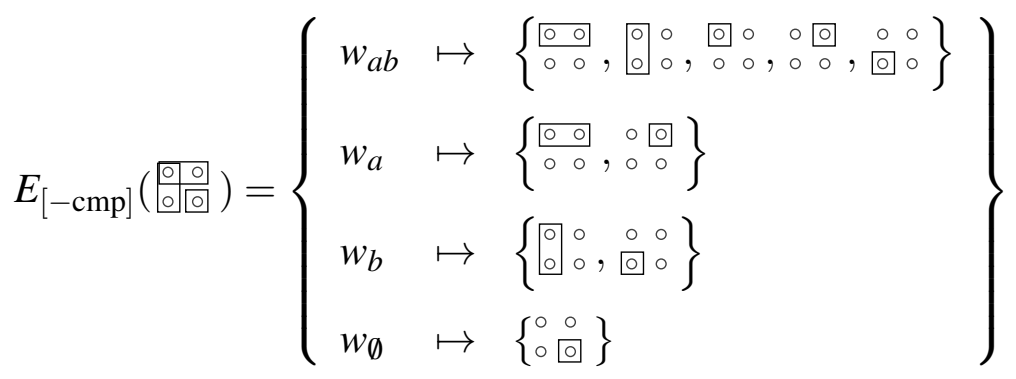

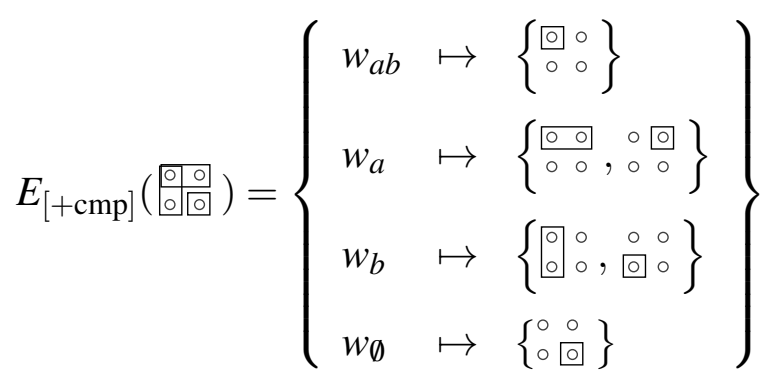

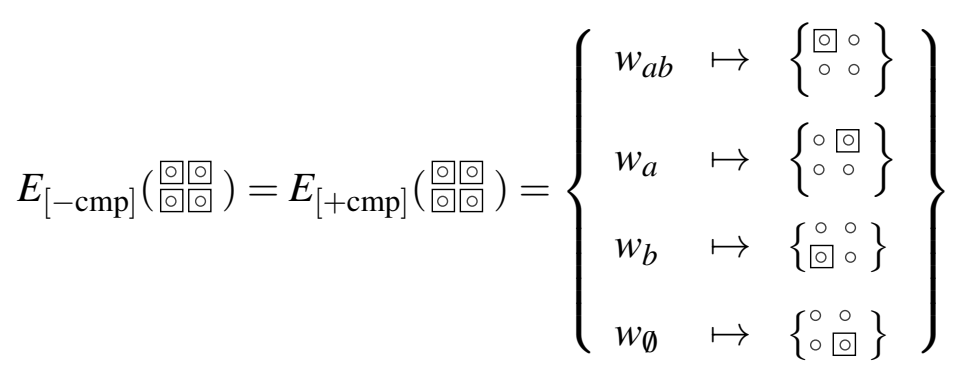


Theiler, Roelofsen \& Aloni

\subsubsection{Embedding verbs}

So far, we laid out an account of interrogative complements. Clearly, what is still missing are the verbs that embed such complements. We consider two examples here, know and be certain. For the treatment of a wider range of verbs in our system, the reader is referred to Theiler, Roelofsen \& Aloni (2016). ${ }^{7}$

In order to formally characterize the meaning of know and be certain, we will employ the notion of an individual $x$ 's information state in a world $w, \sigma_{x}^{w}$, under which we understand the set of worlds compatible with what $x$ takes herself to know in $w$. We assume, as is commonplace in doxastic logic, that $\sigma_{x}^{w}$ is always consistent.

The meanings of know and be certain can be defined as relations between the information state of their subject and the meaning of their complement.

$$
\begin{aligned}
& \text { know }^{\prime} \quad:=\lambda f_{\langle s, T\rangle} \cdot \lambda x . \lambda p . \forall w \in p: \sigma_{x}^{w} \in f(w) \\
& \text { be certain' }^{\prime}:=\lambda f_{\langle s, T\rangle} \cdot \lambda x . \lambda p . \forall w \in p: \forall v \in \sigma_{x}^{w}: \sigma_{x}^{w} \in f(v)
\end{aligned}
$$

Both know' and be certain' take a function $f$ from worlds to sets of propositions and an individual $x$ as arguments, and yield a set of propositions. For us, $f$ is the meaning of an interrogative complement, i.e., a function from worlds to truthful resolutions of the complement. Then, know' yields the set of propositions $p$ such that for every world $w \in p$ the information state of $x$ in $w$ exactly matches one of the truthful resolutions in $f(w)$.

The lexical entry of be certain, which is strongly inspired by Uegaki's (2015) treatment of this verb, differs from the entry of know only in an additional layer of universal quantification over worlds: it is required that $\sigma_{x}^{w}$ is a truthful resolution in all worlds $v \in \sigma_{x}^{w}$. Crucially, since $\sigma_{x}^{w}$ doesn't necessarily contain the world of evaluation $w, \sigma_{x}^{w}$ doesn't necessarily have to be a truthful resolution in $w$. Rather, it suffices if all the worlds $v$ compatible with what $x$ takes herself to know are such that $\sigma_{x}^{w}$ is a truthful resolution in $v$. This means, be certain is sensitive only to the subject's information state and not to the state of affairs in the world of evaluation. We will discuss the consequences this has in Section 3.1.4 and Section 3.2. ${ }^{8}$

Note that our treatment of know differs from standard accounts of this verb. Standardly, it is required that $\sigma_{x}^{w}$ is a subset of $f(w)$, whereas for us $\sigma_{x}^{w}$ has to be an element of $f(w)$ - and as we will see in Section 3.1.4, this is instrumental in accounting for FA sensitivity.

7 Our treatment of know builds on that in inquisitive epistemic logic (Ciardelli \& Roelofsen 2015; Ciardelli 2016). There, however, know is taken to be sensitive to the resolutions of its complement, rather than the truthful resolutions. As a result, FA-sensitivity isn't captured.

8 More generally, know belongs to the class of extensional verbs, while be certain belongs to the class of intensional verbs (Groenendijk \& Stokhof 1984). For a more detailed discussion of this distinction and its connection to FA sensitvity and veridicality, the reader is referred to Theiler et al. (2016). 
Truthful resolutions

\subsubsection{Back to strong exhaustivity, weak exhaustivity and mention-some}

Our account so far includes two parameters, namely exhaustivity, which is a parameter of the nucleus meaning, and completeness, which is a parameter of the $E$ operator. What we still need to do, however, is to relate these parameters to the customary three-way distinction between MS, WE and SE.

The MS reading can be regarded as non-complete and non-exhaustive, the WE reading as complete and non-exhaustive, and the SE reading as complete and exhaustive. This means we can derive the MS reading by letting $E_{[-\mathrm{cmp}]}$ apply to a [-exh] nucleus meaning, cf. (6) above. The WE reading results from $E_{[+\mathrm{cmp}]}$ applying to a [-exh] nucleus meaning, cf. (7) above. Finally, the SE reading can be obtained by having either $E_{[+\mathrm{cmp}]}$ or $E_{[-\mathrm{cmp}]}$ apply to a [+exh] nucleus meaning; whether we take the complete or non-complete version of $E$ is immaterial, cf. (8) above. The readings generated by the different configurations are summarized in the following table.

\begin{tabular}{c|cc} 
& nucleus $_{[-\mathrm{exh}]}$ & nucleus $_{[+\mathrm{exh}]}$ \\
\hline$E_{[-\mathrm{cmp}]}$ & $\mathrm{MS}$ & $\mathrm{SE}$ \\
$E_{[+\mathrm{cmp}]}$ & $\mathrm{WE}$ & $\mathrm{SE}$
\end{tabular}

\subsubsection{False answer sensitivity}

As argued in Section 1.2, an empirically successful account of interrogative complements will have to implement FA sensitivity across all levels of exhaustivity. Let us check whether our analysis lives up to this requirement.

MS readings. We begin with George's scenario, where an MS example is used. Recall that in the actual world $w_{0}$ only Newstopia sells Italian newspapers. Janna and Rupert know that Newstopia sells Italian newspapers. Additionally, Rupert falsely believes that also Paperworld sells such newspapers. Janna has no beliefs about Paperworld. Then, under an MS reading, (11) is judged true, and (12) is judged false.

(11) Janna knows where one can buy an Italian newspaper.

(12) Rupert knows where one can buy an Italian newspaper.

This is indeed what we predict. To see why, assume the above complements each involve $E_{[-\mathrm{cmp}]}$ and the nuclei receive a [-exh] interpretation, resulting in MS readings. Let $P$ be the nucleus meaning. Observe that $P$ contains one true alternative, namely the proposition that one can buy an Italian newspaper at Newstopia, and two false alternatives, namely the proposition that one can buy an Italian newspaper at Paperworld and the proposition that one can buy such a newspaper at neither 
place. Janna's information state $\sigma_{j}^{w_{0}}$ is a truthful resolution of the complement since it is a consistent resolution of $P$ and does not entail any false alternatives in $P$, while Rupert's information state $\sigma_{r}^{w_{0}}$ is not a truthful resolution of the complement since it entails a false alternative in $P$. Thus, (11) comes out as true because $\sigma_{j}^{w_{0}} \in$ $E_{[-\mathrm{cmp}]}(P)\left(w_{0}\right)$, while (12) comes out as false because $\sigma_{r}^{w_{0}} \notin E_{[-\mathrm{cmp}]}(P)\left(w_{0}\right)$.

WE readings. For WE readings, FA sensitivity arises from the same mechanism as for MS readings, for the following reason. To derive a WE reading for (12), the complement needs to include $E_{[+\mathrm{cmp}]}$ instead of $E_{[-\mathrm{cmp}]}$. It is easy to see that any proposition $p$ that doesn't count as a truthful resolution on the MS reading won't count as one on the WE reading either, since, if $p \notin E_{[-\mathrm{cmp}]}(P)\left(w_{0}\right)$, then also $p \notin E_{[+\mathrm{cmp}]}(P)\left(w_{0}\right)$.

SE readings. For an SE reading, finally, assume that the nucleus in (12) receives a [+exh] interpretation. The alternatives in the nucleus meaning $P$ thus form a partition of the logical space s.t. all worlds in a partition cell agree on the places where one can buy an Italian newspaper. That is, $P$ contains one true alternative, namely the proposition that Newstopia is the only place where one can buy an Italian newspaper, and three false alternatives, namely the propositions that Paperworld is the only place where one can buy an Italian newspaper, that both Newtopia and Paperworld sell such newspapers, and that neither Newstopia nor Paperworld sell them. Any consistent resolution that entails the true alternative will be a truthful resolution, while any proposition that entails one of the false alternatives won't come out as a truthful resolution. The latter applies to Rupert's information state $\sigma_{r}^{w_{0}}$.

Lack of FA sensitivity with be certain. In contrast to know, be certain doesn't exhibit FA sensitivity, as evidenced by the fact that (13) is judged true even if Rupert has false beliefs about the availability of Italian newspapers.

(13) Rupert is certain where one can buy an Italian newspaper.

Our account predicts this lack of FA sensitivity. ${ }^{9}$ Due to the world shift in its lexical entry, be certain is insensitive to whether a resolution is truthful in the world of evaluation. More concretely, the entry for be certain in (10) predicts (13) to be true even if Rupert wrongly believes that Paperworld sells Italian newspapers, because in all worlds in $\sigma_{r}^{w}$ Paperworld indeed sells Italian newspapers, meaning that in these worlds $\sigma_{r}^{w}$ is a truthful resolution. ${ }^{10}$

9 In fact, this lack of FA sensitivity is predicted for all intensional verbs (cf. Fn. 8), as shown in Theiler et al. (2016). That is, extensionality is a necessary condition for FA sensitivity.

10 It has further been observed that complements embedded under be certain only allow a SE reading. Due to the universal quantification over worlds in $\sigma_{x}^{w}$, Uegaki's (2015) and our entry capture this fact. For a more detailed discussion of this prediction, see Theiler et al. (2016). 
This concludes our treatment of interrogative complements. We proposed an account that captures the FA sensitivity exhibited by know across all levels of exhaustivity, while correctly predicting a lack of FA sensitivity for be certain.

\subsection{Declarative complements}

Our account can also be applied to declarative complements-no modifications needed. Here, we focus on some specific predictions for such complements, to do with downward closedness, veridicality implications and selectional restrictions.

Downward closedness of declarative complement meanings. As we have seen in Section 3.1.1, when $E$ applies to an interrogative nucleus, the resulting set of truthful resolutions typically exhibits a restricted form of downward closedness. If $E$ applies to a declarative nucleus, on the other hand, the resulting set of truthful resolutions is fully downward closed modulo containing $\emptyset$. To see why, recall that the meaning of a declarative nucleus is a set of propositions $P$ with a single alternative $q$. For every world $w$, we can distinguish two cases: $w \notin q$ or $w \in q$. In the first case, we get alt ${ }_{w}(P)=\emptyset$ and thus also $E(P)(w)=\emptyset$. That is, the set of truthful resolutions is simply empty. In the second case, if $w \in q$, we find that alt ${ }_{w}^{*}(P)=\emptyset$. Now, the truthfulness condition requires that a truthful resolution doesn't entail any alternative in alt $_{w}^{*}(P)$. However, since alt ${ }_{w}^{*}(P)=\emptyset$, this condition is trivially satisfied. Thus, if $E$ applies to a declarative, either the set of truthful resolutions is empty or the truthfulness condition is vacuous. As a consequence, the sets of truthful resolutions are fully downward closed modulo containing $\emptyset$.

Veridicality implications. Empirically, we find that know triggers a veridicality implication when taking a declarative complement:

John knows that Mary called.

$\therefore$ Mary called.

To see how our account captures this fact, assume that in $w$ Mary didn't call. Let $P$ be the meaning of the declarative nucleus in (14). Then, $P$ contains a single alternative $q$ s.t. $w \notin q$ and $q$ is entailed by all resolutions of $P$. This means, $E(P)(w)=\emptyset$. So, John's information state cannot be an element of $E(P)(w)$, and the premise in (14) comes out as false. Conversely, John knows that Mary called can only be true in worlds where Mary called—which amounts to a veridicality implication. ${ }^{11}$

11 In the case of know, the veridicality implication is actually a factivity presupposition. For reasons discussed in Theiler et al. (2016), we let the presuppositional nature of this implication be determined by know and not by $E$. A suitable presuppositional entry for know is the following.

(i) $\quad \mathrm{know}^{\prime}=\lambda f_{\langle s, T\rangle} \cdot \lambda x \cdot \lambda p . \forall w \in p . f(w) \neq \emptyset . \forall w \in p: \sigma_{x}^{w} \in f(w)$ 
In contrast to know, be certain does not trigger a veridicality implication:

John is certain that Mary called.

$\%$ Mary called.

This fact is captured as well. Due to the world shift in the semantics of be certain and the fact that declarative complement meanings are fully downward-closed, John is certain that Mary called comes out as true in any world $w$ such that $\sigma_{j}^{w}$ entails the proposition that Mary called; it need not be the case that Mary indeed called in $w$.

Selectional restrictions. The distribution of declarative and interrogative complement clauses is restricted. In particular, apart from responsive verbs like know, there are also anti-rogative verbs like believe that only embed declarative complements and rogative verbs like wonder that only embed interrogative complements.

a. Mary wonder/*believes who called.

b. John *wonders/believes that Mary called.

One strategy to account for these data is to assume that declarative and interrogative complements differ in semantic type, and that verbs like believe only take complements of one type while verbs like wonder only take complements of the other type. This strategy is not available to us since on our account both kinds of complements have the same type. What we can try to do instead, however, is derive the distributional differences from the semantic properties of the complements and embedding verbs. In fact, seeing that a type-based account is a mere reformulation of the selectional restrictions in terms of semantic types, this alternative strategy holds more explanatory potential. Here, we only provide a brief sketch of the approach; for more detail we refer to Theiler et al. (2016).

We assume that anti-rogative verbs operate purely on the informative content of their complements. ${ }^{12}$ If they take a complement with trivial informative content, then the resulting statement is not contingent, i.e., either a contradiction or a tautology. Now, as discussed in Section 2.2, the informative content of interrogative complements is always trivial. Our account predicts that, if an anti-rogative verb combines with an interrogative complement, then the result is bound to be tautological or contradictory. This systematic non-contingency can be taken to explain why anti-rogative verbs don't license interrogative complements (see Gajewski 2002).

For example, (17) corresponds to a classical Hintikkan treatment of believe (Hintikka 1962). An individual $x$ believes a complement in $w$ exactly if $x$ 's information

12 For certain anti-rogative verbs, this assumption is very natural, e.g., for be true, be false and believe. For these verbs, our account has more explanatory strength than one which is based on a stipulated difference in semantic type. In general, however, to give an explanation that applies to the full range of anti-rogative verbs, one would like to have an independent reason for why all of these verbs should be insensitive to inquisitive content. We leave this for future work. 
state $\sigma_{x}^{w}$ is a subset of the complement's informative content. Since $w$ is contained in the informative content of a complement $f$ just in case $f(w)$ is non-empty, this translates to the following lexical entry.

$$
\text { believe }^{\prime}:=\lambda f_{\langle s, T\rangle} \cdot \lambda x . \lambda p . \forall w \in p: \forall v \in \sigma_{x}^{w}: f(v) \neq \emptyset
$$

To see how the selectional restrictions are accounted for, suppose that believe' combines with an interrogative complement $f$. Then there exists no $v$ such that $f(v)=\emptyset$. Hence, we find that believe $(f)(x)$ is a tautology.

Analogously, we assume that rogative verbs operate on the inquisitive content of their complement and therefore need a complement with non-trivial inquisitive content in order to produce contingent statements. Since declarative complements are always non-inquisitive, this means that rogative verbs taking declarative complements systematically result in non-contingent statements, which again we take to explain the ungrammaticality of such constructions.

\section{Conclusion}

We have proposed an account of interrogative and declarative complements in which the meaning of either kind of complement is a function that maps every world $w$ to the set of truthful resolutions of the issue expressed by the complement in $w$. This kind of meaning representation is fine-grained enough to allow for a treatment of embedding verbs that assumes only a single lexical entry for the declarative and the interrogative embedding use of these verbs. This single entry can uniformly apply to both kinds of complements, with the relevant differences in meaning arising exclusively from the semantic properties of the complement. In particular, with interrogative complements, the proposed semantics derives FA sensitivity, with declarative complements, it gives rise to veridicality implications.

Our approach provides us with a unifying perspective on FA sensitivity, invoking the same mechanism across all levels of exhaustivity. As a result, correct predictions are made for George's (2013) scenario, which represents a challenge for the widespread reductive account of responsive verbs like know.

\section{References}

Champollion, Lucas, Ivano Ciardelli \& Floris Roelofsen. 2015. Some questions in typed inquisitive semantics. Talk at workshop on Questions in Logic and Semantics, Amsterdam, handout available at illc.uva.nl/inquisitivesemantics/ workshops/inqturn-closing-event-2015.

Ciardelli, Ivano. 2016. Questions in logic: Institute for Logic, Language and Computation, University of Amsterdam PhD dissertation. 
Ciardelli, Ivano, Jeroen Groenendijk \& Floris Roelofsen. 2013. Inquisitive semantics: A new notion of meaning. Language and Linguistics Compass 7(9). 459-476.

Ciardelli, Ivano, Jeroen Groenendijk \& Floris Roelofsen. 2015. Inquisitive semantics. European Summer School in Logic, Language and Information lecture notes, available via www.illc.uva.nl/inquisitivesemantics.

Ciardelli, Ivano \& Floris Roelofsen. 2015. Inquisitive dynamic epistemic logic. Synthese 192(6). 1643-1687.

Ciardelli, Ivano, Floris Roelofsen \& Nadine Theiler. 2016. Composing alternatives. To appear in Linguistics and Philosophy.

Cremers, Alexandre. 2016. On the semantics of embedded questions: Ecole Normale Superieure, Paris PhD dissertation.

Cremers, Alexandre \& Emmanuel Chemla. 2016. A psycholinguistic study of the exhaustive readings of embedded questions. Journal of Semantics 33(1). 49-85.

Gajewski, Jon. 2002. L-analyticity and natural language. Manuscript, MIT.

George, Benjamin Ross. 2011. Question embedding and the semantics of answers: University of California, Los Angeles PhD dissertation.

George, Benjamin Ross. 2013. Knowing-wh, mention-some readings, and nonreducibility. Thought: A Journal of Philosophy 2(2). 166-177.

Groenendijk, Jeroen \& Martin Stokhof. 1984. Studies on the semantics of questions and the pragmatics of answers: University of Amsterdam $\mathrm{PhD}$ dissertation.

Hintikka, Jaakko. 1962. Knowledge and Belief: an Introduction to the Logic of the Two Notions. Cornell University Press.

Karttunen, Lauri. 1977. Syntax and semantics of questions. Linguistics and Philosophy 1. 3-44.

Klinedinst, Nathan \& Daniel Rothschild. 2011. Exhaustivity in questions with non-factives. Semantics and Pragmatics 4(2). 1-23.

Spector, Benjamin. 2005. Exhaustive interpretations: What to say and what not to say. Unpublished paper presented at the LSA workshop on Context and Content. Spector, Benjamin \& Paul Egré. 2015. A uniform semantics for embedded interrogatives: An answer, not necessarily the answer. Synthese 192(6). 1729-1784.

Theiler, Nadine. 2014. A multitude of answers: Embedded questions in typed inquisitive semantics. University of Amsterdam, MSc thesis.

Theiler, Nadine, Floris Roelofsen \& Maria Aloni. 2016. A truthful resolution semantics for declarative and interrogative complements. Manuscript, ILLC, University of Amsterdam.

Uegaki, Wataru. 2015. Interpreting questions under attitudes: Massachusetts Institute of Technology $\mathrm{PhD}$ dissertation. 
Truthful resolutions

Nadine Theiler

Floris Roelofsen

Science Park 107

Science Park 107

1098 XG Amsterdam

1098 XG Amsterdam

nadine.theiler.87@gmail.com

floris.roelofsen@gmail.com

Maria Aloni

Science Park 107

1098 XG Amsterdam

m.d.aloni@uva.nl 